RLEINE TEXTE FƯR VORLESUNGEN UND UBUNGEN HERAUSGEGEBEN VON HANS LIETZMANN 104

\title{
DIE \\ RHEINISCH-WESTFÄLISCHE KIRCHENORDNUNG
}

\author{
HERAUSGEGEBEN
}

VON

D. ALFRED UCKELEY

PROFESSOR DER PRAKT. THEOLOGIE

\section{BON N}

A. MARCUS UND E. WEBER'S VERLAG 1912 